\title{
Comparison of endogenous and overexpressed MyoD shows enhanced binding of physiologically bound sites
}

Zizhen $\mathrm{Yao}^{1 \dagger}{ }^{1 \dagger}$ Abraham P Fong ${ }^{2,4 \dagger}$, Yi Cao ${ }^{3}$, Walter L Ruzzo ${ }^{5}$, Robert C Gentleman ${ }^{3}$ and Stephen J Tapscott T, $^{1,6^{*}}$

\begin{abstract}
Background: Transcription factor overexpression is common in biological experiments and transcription factor amplification is associated with many cancers, yet few studies have directly compared the DNA-binding profiles of endogenous versus overexpressed transcription factors.

Methods: We analyzed MyoD ChIP-seq data from C2C12 mouse myotubes, primary mouse myotubes, and mouse fibroblasts differentiated into muscle cells by overexpression of MyoD and compared the genome-wide binding profiles and binding site characteristics of endogenous and overexpressed MyoD.

Results: Overexpressed MyoD bound to the same sites occupied by endogenous MyoD and possessed the same E-box sequence preference and co-factor site enrichments, and did not bind to new sites with distinct characteristics.

Conclusions: Our data demonstrate a robust fidelity of transcription factor binding sites over a range of expression levels and that increased amounts of transcription factor increase the binding at physiologically bound sites.
\end{abstract}

Keywords: Transcription factor, Overexpressed, MyoD, c-Myc, ChIP-seq

\section{Background}

The biological sciences have always relied on model systems to test specific hypotheses, and the general validity of each model system is constantly subject to vigorous debate. This is particularly true when transcription factors are overexpressed in cells, and the investigator(s) extrapolate their findings to the function of the endogenous factor. Although overexpression studies have yielded many significant advances in our understanding of cell biology, their validity is routinely challenged, particularly in manuscript and grant reviews. Intuitively this skepticism is justified. Biochemistry predicts that higher factor concentrations will drive non-physiological protein interactions or, in the case of transcription factors, DNA binding. Therefore, it is often asserted that overexpression of a transcription factor will not accurately reflect the function of that factor at physiologic levels of expression or, at a minimum, that there is no

\footnotetext{
* Correspondence: stapscot@fhcrc.org

${ }^{\dagger}$ Equal contributors

${ }^{1}$ Human Biology Division, Seattle, WA, USA

${ }^{2}$ Clinical Research Division, Fred Hutchinson Cancer Research Center, 1100

Fairview Avenue North, Seattle, WA 98109, USA

Full list of author information is available at the end of the article
}

basis to assume that it does. As logical as this assertion may seem, there is very little experimental evidence to support or refute it, possibly because genome-wide factor binding and transcriptional activation have only recently been possible to assess. Yet, understanding the functional consequences of transcription factor overexpression is very important in cancer cell biology where gene amplifications, such as $N-M Y C$ amplification in neuroblastomas, promote tumor progression.

Previously we reported the genome-wide binding of endogenous MyoD in mouse muscle cells and compared that to exogenously expressed MyoD in mouse embryonic fibroblasts (MEFs) transduced with a MyoD expressing retrovirus [1]. The transduced MEFs had levels of MyoD protein very similar to the endogenous $\mathrm{MyoD}$ and showed a very similar binding profile. Here we compare the genome-wide binding of endogenous MyoD in mouse skeletal muscle cells with highly overexpressed MyoD in MEFs to determine whether overexpression qualitatively alters the binding profile. We find that the overexpressed MyoD binds to the same sites as endogenous MyoD and does not demonstrate binding to novel regions or motifs. 
Our study shows that overexpression of MyoD accurately identifies sites bound by endogenous MyoD, suggesting an intrinsic biological robustness for varying levels of transcription factor in a cell.

\section{Methods \\ ChIP-seq}

ChIP was performed as previously described [1,2]. ChIP samples were prepared for sequencing per the Illumina Sample Preparation protocol with two modifications: (1) DNA fragments of 150-300 bp were selected at the gelselection step; (2) 21 cycles of PCR were performed at the amplification step instead of 18 . For the control samples, untransduced MEFs derived from $\mathrm{Myod}^{-/-} / \mathrm{Myf5}^{-/-}$ mice were ChIPed with MYOD antibody, and mouse myotubes were ChIPed with preimmune serum.

\section{MyoD lentivirus}

cDNA for Myod was cloned into the GFP locus of the pRRL.SIN.cPPT.PGK-GFP.WPRE lentiviral backbone (Addgene), with expression thus driven by the Pgk promoter. Replication-incompetent lentiviral particles were packaged in 293T cells by the Fred Hutchinson Cancer Research Center Lentivirus Core Facility. MEFs were transduced in DMEM containing polybrene at $8 \mu \mathrm{g} / \mathrm{ml}$. After $24 \mathrm{~h}$, media were replaced; cells were switched to differentiation media (1\% heat inactivated horse serum, $10 \mu \mathrm{g} / \mathrm{ml}$ insulin, $10 \mu \mathrm{g} / \mathrm{ml}$ transferrin) $48 \mathrm{~h}$ after infection and harvested 36-40 h later. ChIP and Western blot were performed with a previously characterized MYOD antibody [3]. Western blot bands were quantified with ImageJ.

\section{ChIP-seq peak calling and significance inference}

Sequences were extracted using the GApipeline software. Reads mapping to the $\mathrm{X}$ and $\mathrm{Y}$ chromosomes were excluded from our analysis. Reads were aligned using MAQ to the mouse genome (mm9). Duplicate sequences were discarded to minimize effects of PCR amplification. Each read was extended in the sequencing orientation to a total of 200 bases to infer the coverage at each genomic position. Peak calling was performed by an in-house developed $\mathrm{R}$ package that models background reads by a negative binomial distribution conditioned on GC content as previously described [1,2,4]. The control ChIP-seq sample was used to eliminate statistically significant peaks likely due to artifact.

\section{Motif analysis}

We used a discriminative de-novo motif discovery tool described previously [2] to find motifs that distinguish foreground and background sequence data sets. To find motifs enriched under ChIP-seq peaks, we selected background sequences using random genomic regions sampled with similar GC content and distance to TSS. We infer a positional weight matrix (PWM) model from an output motif using an iterative expectation-maximization (EM) refinement process, which is similar to MEME [5].

\section{ChIP-seq sample comparison}

The scatter plot of the MyoD peak heights in the two samples (endogenous MyoD and lenti-MyoD) indicated a strong correlation (Pearson correlation 0.52 with asinh transformation). Nevertheless, due to the different origins of samples, the overall variation between the two was still far greater than their respective technical replicates (data not shown). Therefore, it was challenging to apply an appropriate statistical null model to capture the stochastic variation between the two systems and to identify exactly the set of peaks that are identical or different between the samples. Therefore, we chose a nonparametric approach by comparing the overlap of peaks at a spectrum of different rank cutoffs in order to outline the global landscape of peak similarity. Cross celltype comparison was performed similarly as previously described [4]. We ranked all peaks by their p-values and group ranks into bins of 5,000 (i.e., the top $5 \mathrm{~K}$ peaks, then the top $10 \mathrm{~K}$ peaks, etc.). Then we computed the fraction of the top $x$ peaks in one sample that overlap with the top $y$ peaks in another sample, where $\mathrm{x}$ and $\mathrm{y}$ vary from $5 \mathrm{~K}$ to $110 \mathrm{~K}$, and $\mathrm{y}$ is equal to or greater than $\mathrm{x}$. To compare the coverage at E-boxes in endogenous and lenti peaks, and to quantify the distribution of peak height ratios between the two samples, we adjusted for the different numbers of total reads by sub-sampling equal numbers of endogenous and lenti reads and recomputed the coverage and peak height at these sites.

\section{Ethical approval}

This study did not directly use vertebrate animals or human subjects and did not require ethical approval.

\section{Results}

Comparison of different MyoD expression levels in the conversion of fibroblasts to skeletal muscle

Mouse embryonic fibroblasts (MEFs) can be converted to skeletal muscle by the forced expression of MyoD. To determine whether overexpression of MyoD can reliably identify biologically relevant binding sites, we compared the binding profile of the endogenous MyoD in mouse muscle cells to the MyoD binding profile in MEFs with exogenously overexpressed MyoD. Transduction of MEFs with a lentivirus expressing MyoD from the Pgk promoter (lenti-MyoD) induced differentiation to skeletal muscle as determined by fusion and expression of myosin heavy chain (Figure 1A). Western analysis demonstrated that lenti-MyoD cells had approximately four-fold higher levels 
of MyoD protein than the endogenous MyoD of $\mathrm{C} 2 \mathrm{C} 12$ myotubes (Figure 1B).

\section{Overexpressed MyoD binds the same sites as endogenous MyoD}

To determine whether overexpressed MyoD can accurately identify sites bound by endogenous MyoD, we compared a ChIP-seq data set obtained from MEFs transduced with lenti-MyoD [4] to our previous ChIPseq data from endogenous MyoD in mouse myotubes [1]. The lenti-MyoD data set had 17.5 million mapped unique reads, and we combined endogenous MyoD ChIP-seq data from mouse C2C12 myotubes (6.5 million reads) and primary differentiated cultured mouse muscle cells (8.5 million reads) to achieve a comparable total 15 million reads for endogenous MyoD because our prior analysis [1] demonstrated a high concordance of peak locations between these two samples. These reads were processed and peaks were identified as described in Methods and Additional file 1: Figure S1A and B. The control ChIP-seq samples ( 18 million pooled reads from pre-immune ChIP in muscle cells, MyoD antisera ChIP in MEFs that do not express MyoD, and beads alone) contained a small number of high peaks (Additional file 1: Figure S1B), which were found at similar locations in all

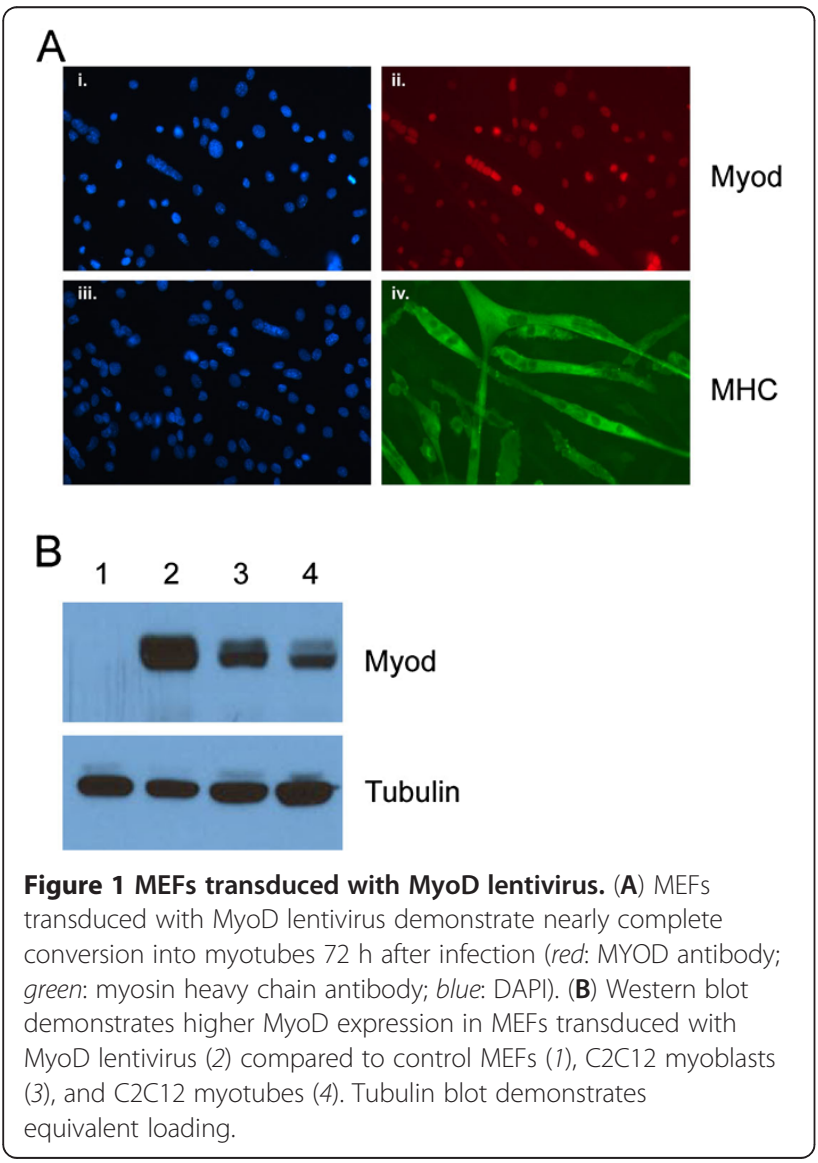

three control ChIP samples (data not shown) and remain of unknown etiology. These non-MyoD peaks were subtracted from the MyoD ChIP-seq data sets.

ChIP-seq for the endogenous MyoD identified $~ 37,000$ peaks at a $p$-value (see Methods) of $10^{-10}$ (read cutoff 20), $\sim 67,000$ peaks at a $p$-value of $10^{-5}$ (read cutoff $\sim 11$ ), and $\sim 117,000$ at a $p$-value of $10^{-3}$ (read cutoff $\sim 8$ ). A similar range of peaks was identified by the overexpressed MyoD but at slightly higher $p$-value thresholds: $\sim 35,000$ ( $\mathrm{p} \sim 10^{-20}$, cutoff $\left.\sim 50\right), \sim 68,000$ ( $\mathrm{p} \sim 10^{-10}$, cutoff $\left.\sim 26\right)$, and $\sim 122,000$ ( $\mathrm{p} \sim 10^{-5}$, cutoff $\sim 14$ ). At a given $p$-value, the overexpressed MyoD had approximately twice the number of peaks compared to the endogenous MyoD. This could either represent higher occupancy of the same sites bound by the endogenous MyoD or a large number of off-target sites bound by the overexpressed MyoD and not bound by the endogenous MyoD.

To accurately compare the similarity, or overlap, of MyoD binding sites in the different samples, we used a non-parametric approach that compared the overlap of peak locations based on the rank order of the peaks in each sample (see Methods). Comparing the top 35,000 peaks bound by endogenous $\mathrm{MyoD}$ and lenti-MyoD, there was a $67 \%$ overlap, and the overlap was similar comparing the top 70,000 or 110,000 peaks for each (Figure 2A). The lack of a complete overlap at each cutoff was largely due to the rank-order of the peaks (based on $\mathrm{p}$-value) rather than distinct binding regions, because $87 \%$ of the top 35,000 lenti-MyoD peaks were represented in the top 70,000 endogenous-MyoD peaks and $93 \%$ in the top 110,000 endogenous-MyoD peaks. Similarly, $85 \%$ and $91 \%$ of the top 35,000 endogenousMyoD peaks were present in the top 70,000 and 110,000 lenti-MyoD peaks, respectively. A more detailed representation of this data is shown in Figure 2B. Therefore, although there were some differences in the rank order of the peaks, the locations were almost the same with greater than $90 \%$ concordance.

\section{Overexpressed MyoD binds the same motifs as endogenous MyoD}

Since MyoD binds as a heterodimer with an E-protein to an E-box containing a CANNTG core sequence, with preference for $\mathrm{GC}$ or $\mathrm{CC}$ as the internal nucleotides $[1,4]$, we next determined whether overexpression of MyoD resulted in binding to a distinct set of low affinity sites or sites that might reflect homodimers or other protein complexes. We used two different approaches to determine the binding site preferences for endogenous and overexpressed MyoD.

First, we ranked all of the approximately 15 million E-boxes in the mouse genome based on their ChIP-seq coverage (see Methods for details of the statistical model) and then binned them by rank as the top 1,000, 1,001-10,000, 
A

\begin{tabular}{|c|c|c|c|}
\hline & \begin{tabular}{|l|} 
Primary.tube \\
$35 \mathrm{~K}$
\end{tabular} & \begin{tabular}{|l|} 
Primary.tube \\
$70 \mathrm{~K}$ \\
\end{tabular} & $\begin{array}{l}\text { Primary.tube } \\
110 \mathrm{~K} \\
\end{array}$ \\
\hline Lenti 35K & $\begin{array}{r}0.67 \\
\end{array}$ & 0.87 & 0.93 \\
\hline Lenti 70K & 0.85 & 0.67 & 0.79 \\
\hline Lenti $110 \mathrm{~K}$ & 0.91 & 0.8 & 0.64 \\
\hline
\end{tabular}

B

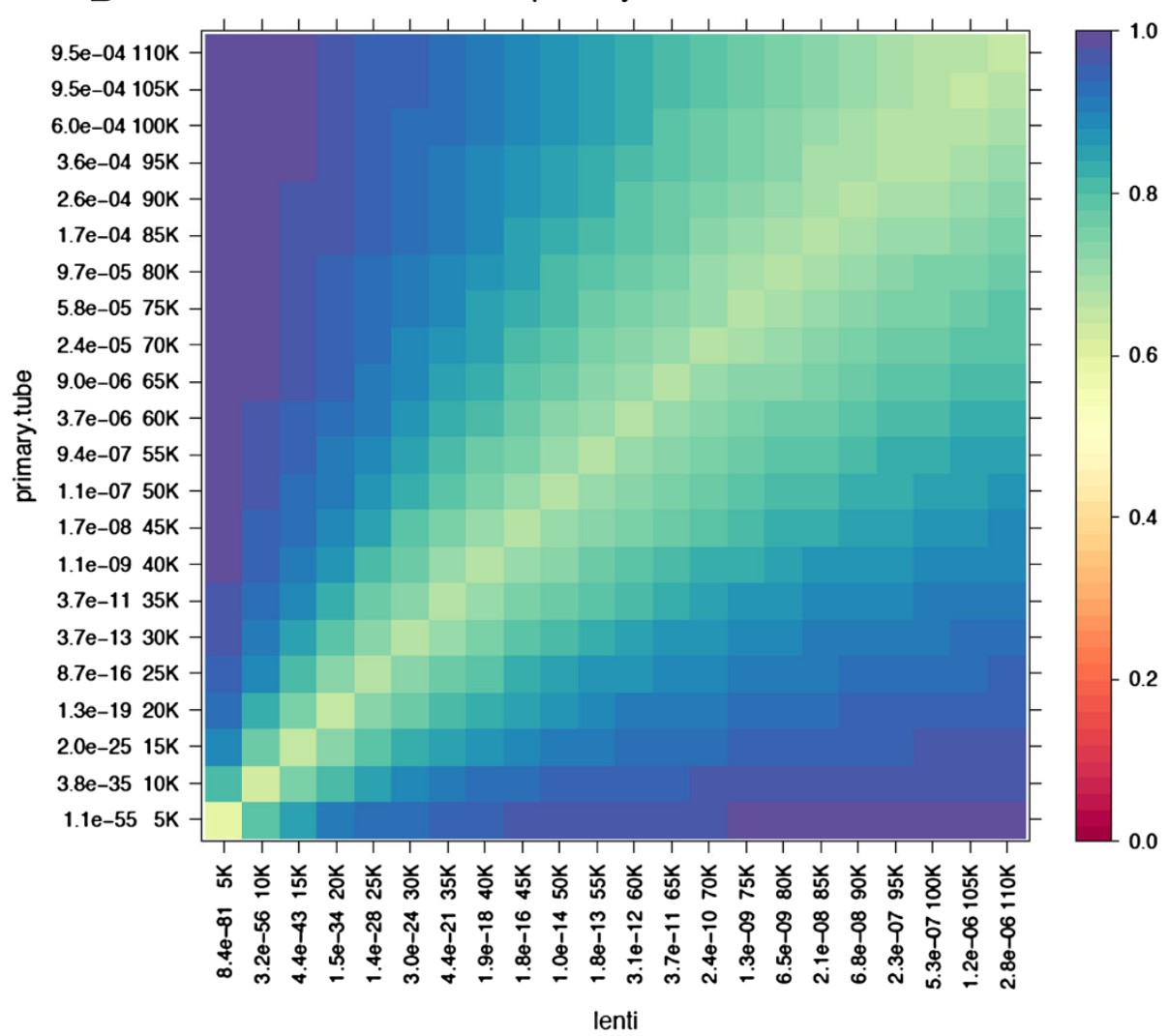

Figure 2 The MyoD binding regions are largely shared between overexpressed MyoD in MEFs and endogenous MyoD. (A) Overlap of lenti-MyoD peaks with endogenous MyoD peaks. To assess the concordance between the two samples, we selected the top $5 \mathrm{~K}$ to $110 \mathrm{~K}$ peaks in each sample based on $p$-value. We calculated the number of overlapping peaks for the top peak sets at various rank cutoffs in both samples and divided this number by the size of the smaller peak set. Specifically, for a cell corresponding to the top $x$ peaks in sample 1 compared to the top y peaks in sample 2, the fraction is computed as the number of overlapping peaks divided by the smaller value of $x$ or $y$. (B) The overlapping fractions were calculated as in A and are plotted with color-coding as specified in the figure. For example, in the cell at the row labeled 5K and column labeled 10K, we plotted the fraction of the top 5K peaks in lenti-MyoD that overlap with the top $10 \mathrm{~K}$ peaks in primary $+\mathrm{C} 2 \mathrm{C} 12$ myotubes.

10,001-100,000, etc. For both endogenous and lenti-MyoD, the CAGCTG and CACCTG E-boxes were enriched for MyoD binding (Figure 3A) in similar proportions within each bin. Therefore, overexpressed MyoD binds a similar distribution of E-box sequences as the endogenous MyoD.

For the second approach to determine whether overexpression of MyoD resulted in binding to a different set of E-boxes, we used a motif discovery algorithm to identify preferred E-box sequences associated with the top 35,000 ranked peaks (ranked on $p$-value), the 35,00170,000 peaks, and the 70,001-110,000 peaks for both endogenous MyoD and overexpressed MyoD (Figure 3B). The identified E-box motifs, and flanking preferences were nearly identical for endogenous MyoD and overexpressed MyoD at different rankings, although the lower ranked peaks had a slightly more degenerate sequence compared to the higher ranked peaks in both groups. Plotting the average position weight matrix (PWM) score for the highest PWM E-box under each peak against the rank of the peaks demonstrates that the average PWM for the low ranked peaks (rank > 85,000) falls off more rapidly for the 


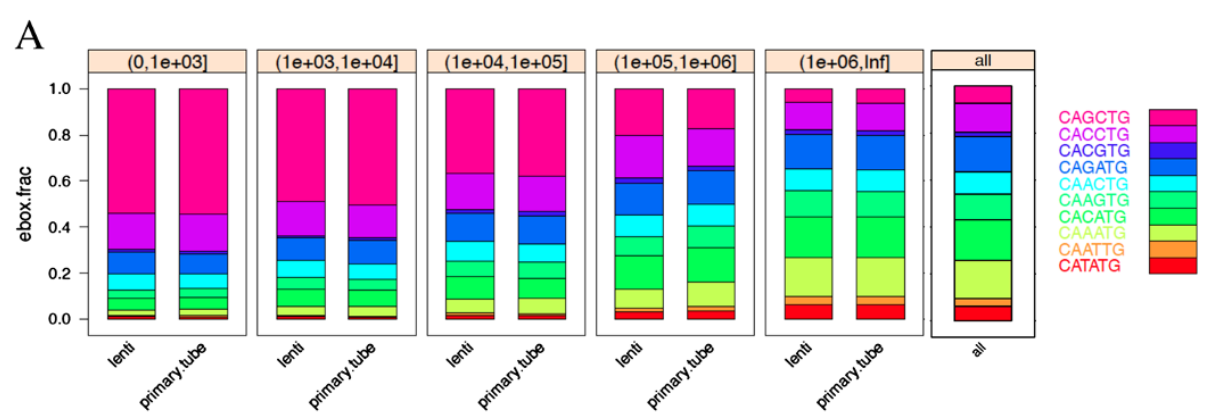

B

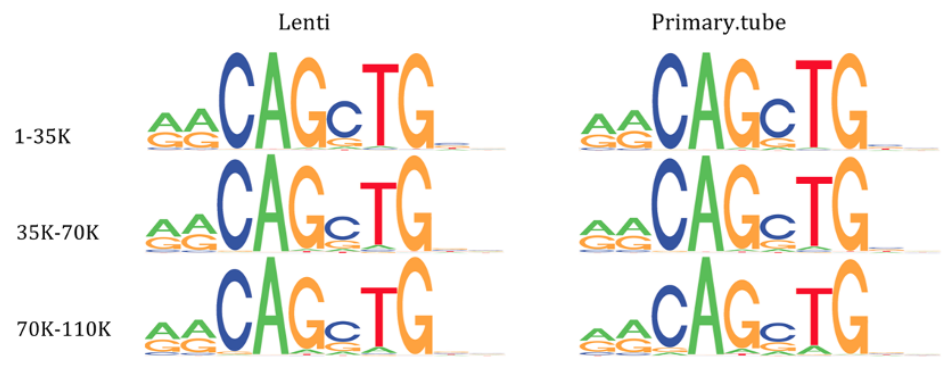

$\mathrm{C}$

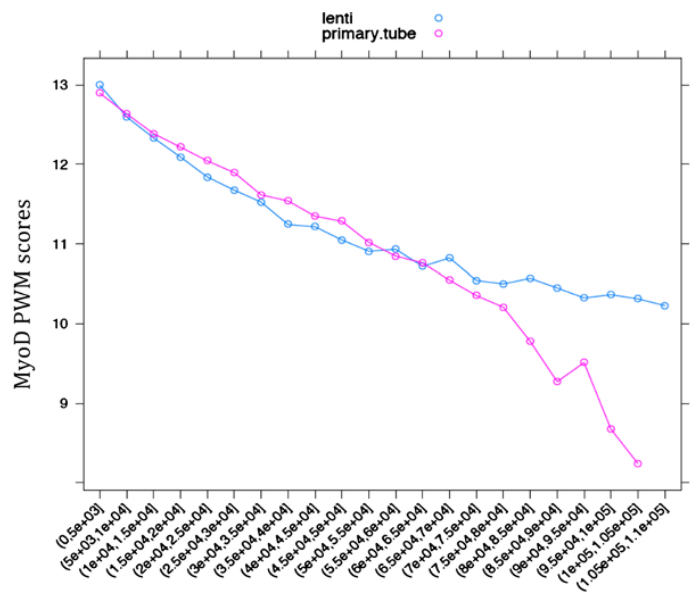

Figure 3 MyoD has a similar E-box preference for both endogenous and overexpressed MyoD. (A) Overexpressed MyoD (lenti) and endogenous MyoD (primary.tube) have similar E-box distributions. We collected all genomic E-boxes (excluding sex chromosomes and those present in the peaks of the control samples) and ranked them based on $p$-values for the read coverage at the E-boxes. We partitioned them into bins of top 1K, top 1001 to 10K, etc., until all E-boxes are included. Within each bin, we calculated the percentage of each type of E-box variant, and plotted the distribution. The background E-box distribution over the entire genome is also included as a reference. (B) MYOD binding sites for overexpressed MyoD (lenti) and endogenous MyoD (primary.tube) share the same sequence preference. We used motif discovery to identify the E-box motif under MyoD bound peaks for the top 35K, the top $35 \mathrm{~K}+1$ to $70 \mathrm{~K}$ peaks, and the top $70 \mathrm{~K}+1$ to $110 \mathrm{~K}$ peaks. The E-box sequence preferences are nearly identical, including within the flanking regions. Motifs in lower ranking peaks tend to have slightly more sequence degeneracy. (C) MyoD E-box average PWM score compared to peak rank. The MyoD PWM is derived from our previous study [4]. Weak peaks tend to have weaker motifs, but the degradation is more gradual for overexpressed MyoD peaks (lenti) beyond the top 67K, suggesting that a subset of noisy low peaks in the endogenous MyoD (primary.tube) is elevated to reasonably strong peaks distinguished from the background. X-axis: the peak rank bins. Y-axis: the average PWM scores for all peaks within the rank bin.

endogenous MyoD compared to the lenti-MyoD (Figure 3C). This likely reflects the difficulty of accurately discriminating weak MyoD binding sites from background reads for the endogenous MyoD, whereas the lenti-MyoD maintains a higher average E-box
PWM score, suggesting that the overexpression of MyoD enhances the ability to discriminate weaker MyoD binding sites from background. Taken together, the data indicate that overexpressed MyoD in MEFs binds to a nearly identical set of sites and E-boxes as 
the endogenous MyoD in mouse muscle cells without a substantial number of off-target sites.

To determine whether overexpressed $\mathrm{MyoD}$ was bound to all of the E-boxes that match the consensus site, we graphed the number of reads over all the RRCAGSTG sites in the mappable genome (Figure 4A), sub-sampling the same number of reads for both the overexpressed and endogenous MyoD ChIP-seq data sets. Despite overexpression in the lenti-MyoD samples, the majority of these high PWM E-boxes had one read or less, similar to the endogenous-MyoD samples, and for both samples fewer than 25\% of these high PWM E-boxes had more than four reads. Therefore, for both the endogenous and overexpressed MyoD, a minor subset of high PWM E-boxes was occupied by MyoD.

Previously, we had measured E-box accessibility in fibroblasts prior to the expression of MyoD by exposing isolated nuclei to the restriction enzyme PvulI, which cleaves at CAGCTG E-boxes [4]. Using these data, we assigned each CAGCTG E-box to one of three groups: relatively inaccessible, moderately accessible, or highly accessible. About $40 \%$ of all highly accessible CAGCTG E-boxes had more than four reads in the endogenous MyoD samples, and slightly over $50 \%$ had four or more reads in the overexpressed MyoD samples; this compares to approximately $15 \%$ for both samples over the relatively inaccessible E-boxes (Figure 4B). However, only about one-third of the highly accessible group had read coverage above the average cutoff for the top $\sim 70,000$ peaks (11 reads for endogenous MyoD and 26 reads for overexpressed MyoD). Therefore, E-box accessibility in the chromatin was a major determinant of MyoD binding, but a large fraction of relatively highly accessible E-boxes with a strong PWM remained unbound by MyoD, indicating that only a subset of accessible E-boxes with a good PWM showed substantial MyoD binding even when MyoD was overexpressed. In this regard, our prior study [4] showed that several sequence motifs were enriched in the region of bound accessible sites (additional E-boxes, higher PWM E-boxes, and a motif similar to a MEIS binding site), indicating that several factors might operate to enhance or stabilize MyoD binding at subsets of accessible sites.

\section{Co-factor motifs and MyoD binding}

Although there was a very high concordance of binding sites for endogenous MyoD in mouse muscle cells and overexpressed MyoD in MEFs, there was some difference in peak rank, as evidenced by a $67 \%$ overlap of the top 35,000 peaks in each set with most of the additional $33 \%$ of peaks present in the other set at lower rank. Since the E-box motifs were similar in both sets and did not apparently account for rank differences, we examined the top 30,000 peaks in each set for co-factor motifs using a de novo motif search strategy (see Methods). The peaks in the mouse muscle cells were enriched in E-box motifs ( $>7$-fold) and had modest enrichment for several other motifs: MEIS (1.6-fold), RUNX (1.3-fold), and AP1 (2.2-fold). Similarly, the peaks in the MEFs with overexpressed MyoD were enriched for E-box motifs (>6-fold), MEIS (1.6-fold), and RUNX (1.4-fold) relative to the background sequence (Additional file 1: Figure S2A). It remains possible that some of the rank differences reflect the relative abundance of co-factors in the different cell backgrounds, but there is not a strong association of a specific factor motif with the MyoD binding sites.

Although there was about 90\% concordance between endogenous and overexpressed MyoD peaks, albeit with some difference in ranking position, approximately $5-7 \%$ of the highly ranked peaks in each set was not represented in the top 110,000 peaks in the other set. Motif analysis of the sequences under the endogenous-only peaks (i.e., ranked in the top 30,000 endogenous peaks but not in the top 110,000 lenti-MyoD peaks) compared to peaks only present in the lenti-MyoD (i.e., ranked in the top 30,000 lenti-MyoD peaks but not in the top 110,000 endogenous MyoD peaks) showed an enrichment of a variant of the RUNX motif (2-fold), a PITXlike motif (4-fold), and a CGNCAG motif (2.7-fold). A similar motif analysis comparing the endogenous-only peaks to shared peaks also revealed the RUNX and PITX-like motifs, albeit at a slightly lower fold enrichment. The comparable analysis to identify motifs in the lenti-only peaks revealed a slight enrichment for E-boxes with non-preferred core sequences (Additional file 1: Figure S2B), indicating that a small number of the lentionly peaks might represent binding to lower affinity sites, possibly driven by the higher amount of MyoD. Therefore, while there might be some contribution of co-factors expressed in the mouse muscle cells that accounts for the small number of endogenous-only peaks, the motif analysis does not identify more than a modest enrichment of the motif for any specific factor, consistent with the finding that the MyoD binding sites in both cells types show over $90 \%$ concordance.

\section{Discussion}

We conclude that overexpression of MyoD can accurately identify endogenous MyoD binding sites. This is true despite the fact that the binding pattern of the endogenous MyoD was determined in skeletal muscle cells (primary myotubes and differentiated $\mathrm{C} 2 \mathrm{C} 12$ cells), whereas the binding sites of the overexpressed MyoD were determined in MEFs. The concordance of binding sites in these two cell types might reflect the ability of MyoD to convert MEFs to skeletal muscle. In this process, MyoD activates the expression of many cofactors that cooperate in a feed-forward circuit with 

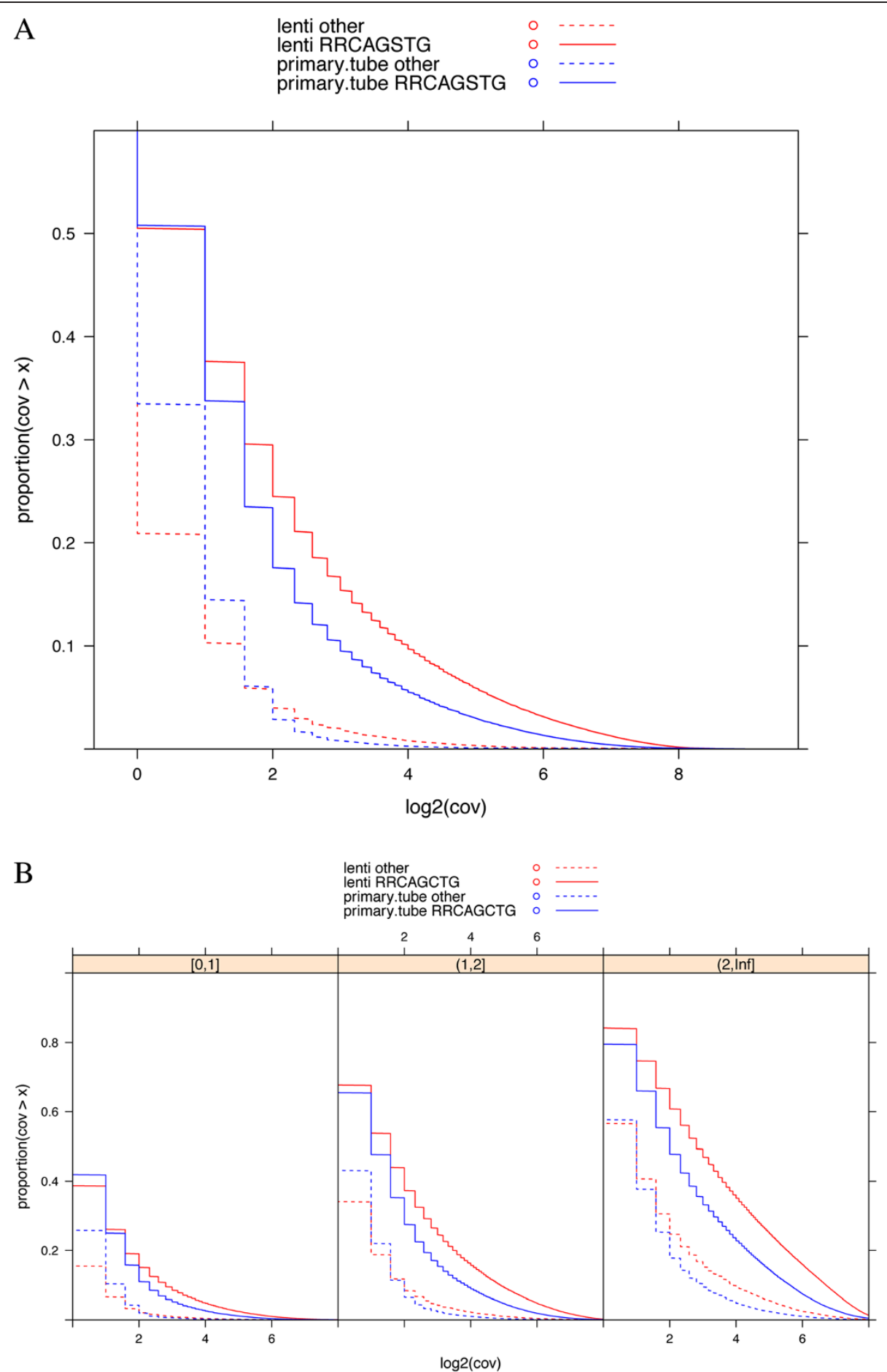

Figure 4 MyoD binds a subset of accessible E-boxes. (A) Coverage distribution over consensus MyoD binding sites RRCAGSTG. X-axis: log2 transformed coverage. Y-axis: the proportion of sites with coverage greater than the given value $\mathrm{x}$. To make read coverage more comparable, we sub-sampled the same number of reads in each group. The distributions of coverage at RRCAGSTG sites in both samples are shown in solid lines. For comparison, E-boxes other than RRCAGSTG sites are shown in dashed lines. Only the E-boxes that are uniquely mapped within a $\pm 200-b p$ window are included. (B) Coverage distribution over E-boxes similar to panel $\mathbf{A}$ but divided into E-boxes showing relatively low accessibility $(0,1]$, moderate accessibility $(1,2]$, or relatively high accessibility $(2, \mathrm{lnf}]$, as previously determined by Pvull accessibility [4]. 
MyoD to orchestrate gene expression [7-9]. Since MyoD can activate its own co-factors, the initial differences in co-factor expression between muscle cells and MEFs might not alter the ultimate binding pattern of MyoD.

In differentiating muscle cells, MyoD binds DNA as a heterodimer with an E-protein. However, in vitro binding studies demonstrate that MyoD can form homodimers and bind E-boxes. Therefore, we had anticipated that overexpressed MyoD might bind as a homodimer because of limiting amounts of E-proteins. Surprisingly, we do not think there is any evidence for homodimer binding. The E-box motif analysis of the endogenous MyoD has asymmetric flanking sequences: RRCAGSTG. In a recent study we have shown that NeuroD2 binds an E-box with similar flanking preferences on one side: RRCAGMTGG [4]. Because both MyoD and NeuroD2 form heterodimers with the same E-proteins, we assume that the flanking $R R$ is determined by the common E-protein partner, and initial binding studies support this conclusion (AP Fong, unpublished data). Since these flanking preferences are maintained at the E-boxes when MyoD is overexpressed, it suggests that the E-protein determined sequence preference is maintained and that MyoD is binding as a heterodimer even when overexpressed. It is possible that the requirement for heterodimer binding prevents offtarget DNA binding by the overexpressed MyoD since the amount of the E-protein dimerization partner would be limiting.

The fact that overexpression of MyoD improved the foreground-to-background signal and permitted site determination at higher p-value stringency suggests that many of the MyoD binding sites might not be occupied 100\% of the time at endogenous levels of MyoD, although this remains speculative since other unknown variables might have affected the ChIP efficiencies or foreground/background read ratios in the different experiments. With these caveats in mind, if the majority of sites are not saturated by physiological levels of MyoD (i.e., not bound by MyoD $100 \%$ of the time) then these would present a large "sink" for the overexpressed MyoD protein, which might further limit ectopic binding. In this regard, it is interesting to note that re-analysis of published c-Myc binding ChIP-seq data [6] under low and high serum conditions that result in an approximately five-fold change in $c-M y c$ mRNA also shows enhanced binding of weakly bound sites with increased c-Myc levels (ZY and SJT, unpublished data). Furthermore, while this manuscript was under review, Lin et al. [10] demonstrated that increased amounts of c-Myc protein resulted in greater saturation of weakly bound cMyc sites near promoters and this was associated with increased gene transcription. Additional studies will be required to determine whether increased MyoD binding at physiologically unsaturated sites has a similar function in enhancing gene transcription. Together these findings suggest that transcription factor overexpression, e.g., induced by gene amplification or other mechanisms in cancers, might have major biological consequences as a result of increased binding at physiologically bound sites.

\section{Conclusions}

Our comparison of genome-wide binding of endogenous MyoD with overexpressed MyoD demonstrated that overexpressed MyoD binds to the same sites as endogenous MyoD and does not demonstrate binding to novel regions or motifs. The samples with overexpressed MyoD showed better foreground-to-background signal and permitted site determination at higher statistical significance, suggesting that increased amounts of transcription factor increased the binding at physiologically bound sites. Overall, our study shows that overexpression of MyoD accurately identifies sites bound by endogenous MyoD and demonstrates an intrinsic biological robustness for varying levels of transcription factor in a cell.

\section{Accession numbers}

ChIP-seq data have been deposited in Gene Expression Omnibus (GEO) under accession number GSE34906 (lentioverepressed MyoD) and in DDBJ Sequence Read Archive (DRA) accession number SRP001761 (endogenous MyoD).

\section{Additional file}

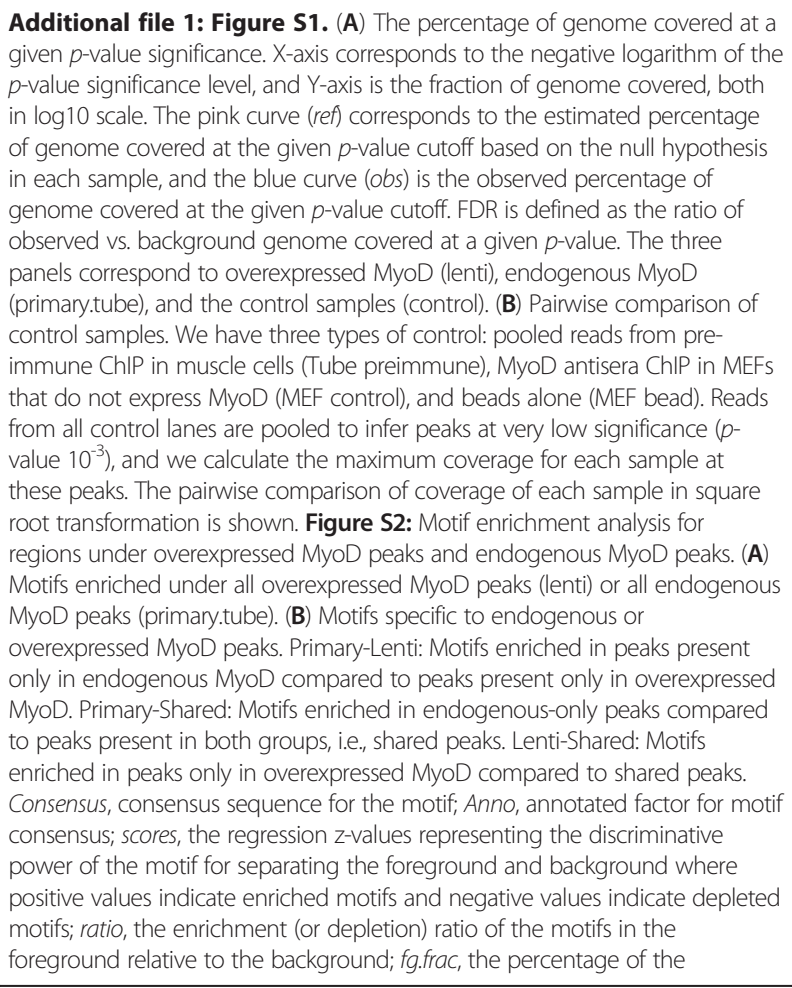

Additional file 1: Figure S1. (A) The percentage of genome covered at a given $p$-value significance. $X$-axis corresponds to the negative logarithm of the $p$-value significance level, and $Y$-axis is the fraction of genome covered, both in log10 scale. The pink curve (ref) corresponds to the estimated percentage of genome covered at the given $p$-value cutoff based on the null hypothesis in each sample, and the blue curve (obs) is the observed percentage of genome covered at the given $p$-value cutoff. FDR is defined as the ratio of observed vs. background genome covered at a given $p$-value. The three (primary.tube), and the control samples (control). (B) Pairwise comparison of control samples. We have three types of control: pooled reads from preimmune ChIP in muscle cells (Tube preimmune), MyoD antisera ChIP in MEFs that do not express MyoD (MEF control), and beads alone (MEF bead). Reads from all control lanes are pooled to infer peaks at very low significance $(p$ value $\left.10^{-3}\right)$, and we calculate the maximum coverage for each sample at these peaks. The pairwise comparison of coverage of each sample in square root transformation is shown. Figure S2: Motif enrichment analysis for regions under overexpressed MyoD peaks and endogenous MyoD peaks. (A) Motifs enriched under all overexpressed MyoD peaks (lenti) or all endogenous MyoD peaks (primary.tube). (B) Motifs specific to endogenous or overexpressed MyoD peaks. Primary-Lenti: Motifs enriched in peaks present only in endogenous MyoD compared to peaks present only in overexpressed MyoD. Primary-Shared: Motifs enriched in endogenous-only peaks compared to peaks present in both groups, i.e., shared peaks. Lenti-Shared: Motifs enriched in peaks only in overexpressed MyoD compared to shared peaks. Consensus, consensus sequence for the motif; Anno, annotated factor for motif positive values indicate enriched motifs and negative values indicate depleted ofifs ratio, the enrichment (or depletion) ratio of the motifs in the 
foreground sequences containing the motif; bg.frac, the percentage of the background sequences containing the motif; logo, the PWM logo.

\section{Abbreviations}

MEFs: Mouse embryonic fibroblasts; lenti-MyoD: MEFs transduced with MyoD lentivirus; PWM: Position weight matrix; EM: Expectation-maximization.

\section{Competing interests}

The authors declare that they have no competing interests.

\section{Authors' contributions}

ZY performed the computational analysis; APF and YC performed the experiments; WLR and RCG provided oversight for the computational analysis; SJT provided oversight for the biological experiments; all authors participated in the experimental design and interpretation. All authors read and approved the final manuscript.

\section{Acknowledgments}

This study was supported by NIH NIAMS R01AR045113; A.P.F was supported by a grant from the University of Washington Child Health Research Center, NIH U5K12HD043376-08; Z.Y. was supported by an Interdisciplinary Training Program grant, T32 CA080416. We thank Mark Biggin for suggesting analysis of saturation and Bruno Amati and Heiko Muller for sharing coverage data from their c-Myc study [6]

\section{Author details}

${ }^{1}$ Human Biology Division, Seattle, WA, USA. ${ }^{2}$ Clinical Research Division, Fred Hutchinson Cancer Research Center, 1100 Fairview Avenue North, Seattle, WA 98109, USA. ${ }^{3}$ Bioinformatics and Computational Biology, Genentech, South San Francisco, CA, USA. ${ }^{4}$ Department of Pediatrics, University of Washington, School of Medicine, Seattle, WA 98105, USA. ${ }^{5}$ Departments of Computer Science and Engineering and Genome Sciences, Seattle, WA, USA. ${ }^{6}$ Department of Neurology, University of Washington, School of Medicine, Seattle, WA 98105, USA.

Received: 2 October 2012 Accepted: 6 March 2013

Published: 8 April 2013

\section{References}

1. Cao Y, Yao Z, Sarkar D, Lawrence M, Sanchez GJ, Parker MH, MacQuarrie KL, Davison J, Morgan MT, Ruzzo WL, et al: Genome-wide MyoD binding in skeletal muscle cells: a potential for broad cellular reprogramming. Dev Cell 2010, 18:662-674.

2. Palii CG, Perez-Iratxeta C, Yao Z, Cao Y, Dai F, Davison J, Atkins H, Allan D, Dilworth FJ, Gentleman R, et al: Differential genomic targeting of the transcription factor TAL1 in alternate haematopoietic lineages. EMBO J 2011, 30:494-509.

3. Tapscott SJ, Davis RL, Thayer MJ, Cheng PF, Weintraub H, Lassar AB: MyoD1: a nuclear phosphoprotein requiring a Myc homology region to convert fibroblasts to myoblasts. Science 1988, 242:405-411.

4. Fong AP, Yao Z, Zhong JW, Cao Y, Ruzzo WL, Gentleman RC, Tapscott SJ: Genetic and Epigenetic Determinants of Neurogenesis and Myogenesis. Dev Cell 2012, 22(4):721-735.

5. Bailey $T L$, Elkan C: The value of prior knowledge in discovering motifs with MEME. Proceedings of International Conference on Intelligent Systems for Molecular Biology; ISMB. Proc Int Conf Intell Syst Mol Biol 1995, 3:21-29.

6. Perna D, Faga G, Verrecchia A, Gorski MM, Barozzi I, Narang V, Khng J, Lim KC, Sung WK, Sanges R, et al: Genome-wide mapping of Myc binding and gene regulation in serum-stimulated fibroblasts. Oncogene 2012, 31:1695-1709.

7. Cao Y, Kumar RM, Penn BH, Berkes CA, Kooperberg C, Boyer LA, Young RA, Tapscott SJ: Global and gene-specific analyses show distinct roles for Myod and Myog at a common set of promoters. EMBO J 2006, 25:502-511.
8. Penn BH, Bergstrom DA, Dilworth FJ, Bengal E, Tapscott SJ: A MyoDgenerated feed-forward circuit temporally patterns gene expression during skeletal muscle differentiation. Genes Dev 2004, 18:2348-2353.

9. Tapscott SJ: The circuitry of a master switch: Myod and the regulation of skeletal muscle gene transcription. Development 2005, 132:2685-2695.

10. Lin CY, Loven J, Rahl PB, Paranal RM, Burge CB, Bradner JE, Lee TI, Young RA: Transcriptional amplification in tumor cells with elevated c-Myc. Cell 2012, 151:56-67.

doi:10.1186/2044-5040-3-8

Cite this article as: Yao et al:: Comparison of endogenous and overexpressed MyoD shows enhanced binding of physiologically bound sites. Skeletal Muscle 2013 3:8.

\section{Submit your next manuscript to BioMed Central and take full advantage of:}

- Convenient online submission

- Thorough peer review

- No space constraints or color figure charges

- Immediate publication on acceptance

- Inclusion in PubMed, CAS, Scopus and Google Scholar

- Research which is freely available for redistribution 